\title{
Comparative study of resveratrol in wines from three main grape regions in China
}

\author{
Yan Cui ${ }^{1, a}$ and Wen Lv ${ }^{2, b}$ \\ ${ }^{1}$ College of Food science and Biological Engineering,Tianjin Agricultural University, Tianjin \\ Engineering and Technology Research Center of Agricultural Products Processing,Tianjin \\ 300384, China \\ ${ }^{2}$ Sino-French joint-venture dynasty winery Itd.300402,China

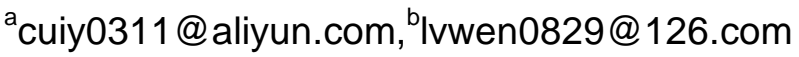

Keywords: Resveratrol, HPLC, Region, Wine

Abstract. In this paper, using high performance liquid chromatography (HPLC), trans resveratrol and trans piceid contents in different style wines (red,white,fresh,aged) from three typical grape regions in China(Hebei Huailai, Ningxia Qingtongxia,Xinjiang Changii) were determined. The role of grape varieties, grape regions and aging practice played in resveratrol contents in wines were discussed. The results showed that total resveratrol content in merlot red wine from Ningxia Qingtongxia was the highest $(6.79 \mathrm{mg} / \mathrm{L})$. The resveratrol contents in merlot wines were higher than those in Cabernet Sauvignon. The resveratrol contents in all the red wine was much higher than in white wines. There was no significant difference in total resveratrol concentrations between fresh wines and aged wines, while trans-piceid contents increased and trans-resveratrol contents decreased during aging process.

\section{Introduction}

Resveratrol (trans-3,4,5- trihydroxystilbene) is a very important phytoalexin produced naturally by some plants in response to bacterial or fungal infections ${ }^{[1]}$. Resveratrol exists naturally in about 700 plants, including mulberry,grape and their derived products, like grape wines. It has two geometric isomers: cis- and trans-, they are either free or bound to glucose as piceid,the $3 \beta$-glucoside of resveratrol. Wine is an excellent source of the trans- form resveratrol,including trans-resveratrol and trans-piceid in Fig.1. Because cis-resveratrol and piceid are very unstable and not easy to be detected due to the photosensitivity. As one of antioxidant polyphenol compounds in wines, it shows a potential health benefit for consumers,known as the "French paradox", Which suggested that red wine consumption might protect people from cardiovascular disease despite high levels of dietary saturated $\mathrm{fat}^{[2]}$.

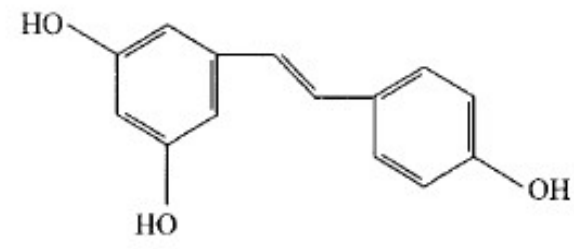

trans-resveratrol

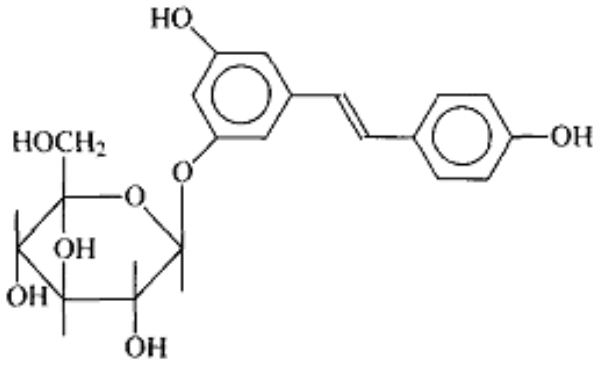

trans-piceid

Fig.1 Molecular structures of two trans- form of resveratrol

Although some previous studies overstated health benefits of resveratrol,there are still some evidences demonstrated that resveratrol have anticancer action, especially to skin and gastrointestinal tumors ${ }^{[3]}$, Resveratrol treatment may prevent the development of mammary tumors in animal models ${ }^{[4]}$. resveratrol has antidiabetic effects by acting against PPARgamma, a pharmacological target for 
diabetes type II. ${ }^{[5]}$ Rsveratrol has antioxidant properties,could prevent premature skin ageing ${ }^{[6]}$. And its neuroprotective effects have been confirmed in several animal model studies. ${ }^{[7]}$

As we known, the contents of resveratrol in wines are as different as grape varietes, origins,climate, vineyard,brewing technics, brewing yeasts and aging and so on. In China, the wines from three main grape regions (Ningxia Qingtongxia, Hebei Huailai and Xinjiang Changji ) performed better and better in recent years and attracted more and more attentions of researchers and brewers. The present paper describes the determination of trans-resveratrol and trans-piceid in 15 wines of three main regions in China using a HPLC based method. The results will provide more informations of resveratrol in different regions wines in China.

\section{Materials and methods}

Samples and chemicals. Fifteen wine different samples were collected respectively from three wineries in the three grape regions in China. Five different wines each winery provided, including one dry white wine Chardonnay and four dry red wines: two Cabernet Sauvignon wines (fresh wine and aging wine aged for 18 months respectively),two Merlot wines (fresh wine and aging wine aged for 18 months respectively), all grapes were from 2012 vintage years, and 10 bottles every sample.

Trans-Resveratrol (>99\%) and trans-piceid (>99\%) were purchased from Tianjin jince fenxi Ltd. methanol (LC-grade) and acetonitrile(LC-grade) were purchased from Tianjin standard technic Ltd.Ultrapure water was purchased from Hangzhou wahaha group ltd. All chemicals used in this study were of HPLC grade or analytical grade.

Pretreatment of wine samples. 50mLof wine sample was taked accurately and then extracted repeatedly for 3 times with $50 \mathrm{~mL}$ of acetic ester, then all the extracted solutions were collected and washed with $3 \%$ of sodium bacarbonate solution and bidistilled water until the solution was neutral, and then evaporated the solution to dryness at $40^{\circ} \mathrm{C}$ with a Rotary Evaporator. After this, the residue was diluted with methanol to $10 \mathrm{~mL}$, and then fitered with a $0.45 \mu \mathrm{m}$ ultrafiltration membrane,finally stored at $4^{\circ} \mathrm{Cfor}$ use.

Analytical methods. Resveratrol contents were determined on a model 1200 Series HPLC from Agilent Technologies coupled with a G1314B UV detector. A ZORBAX SB-C18 column $(4.6 \times 150 \mathrm{~mm})$ was used for the HPLC analysis and was operated at room temperature. acetonitrile-water(40: 60 ) were used as mobile phase at a flow rate of $0.6 \mathrm{~mL} / \mathrm{min}$ and the wavelength of $306 \mathrm{~nm}$, the injection volume was $20 \mathrm{uL}$.

All the results were average resveratrol amount of five detections.

\section{Results and Discussion}

Accurate determination of resveratrol contents was carried out according to the method mentioned above. Trans-resveratrol and trans-piceid were identified by the retention time and absorbtion spectra of standard chemicals. All the wine samples were analysized and the resluts listed in table 1 .

From the table1, The concentration of trans-resveratrol of all wines ranged from $0.21-2.87 \mathrm{mg} / \mathrm{L}$, that of trans-piceid spaned between $0-3.92 \mathrm{mg} / \mathrm{L}$, the total resveratrol reached to $6.79 \mathrm{mg} / \mathrm{L}$ in merlot dry red fresh wine from Ningxia. There were obvious difference in resveratrol contents among the wines. And trans-piceid was higher than trans-resveratrol in most wines. The highest concentration of trans-resveratrol was $2.87 \mathrm{mg} / \mathrm{L}$ in melot wines from Ningxia Qingtongxia grape region. The highest amount of trans-piceid was $3.92 \mathrm{mg} / \mathrm{L}$ in the same wines. In red wines, the least contents of total resveratrol was $4.45 \mathrm{mg} / \mathrm{L}$ in aged Cabernet Sauvignon wines from Xinjiang Changji. While in white wines, Trans-piceid has not been detected in both Hebei and Ningxia Chardonnay wines, the highest resveratrol amount reached to only $0.84 \mathrm{mg} / \mathrm{L}$ from Ningxia Chardonnay wine, no big difference of resveratrol contents was found in white wines from the three regions. 
Table 1: Resveratrol contents of the different wines from three grape regions

\begin{tabular}{|c|c|c|c|c|c|c|c|c|c|c|}
\hline \multirow{3}{*}{$\begin{array}{l}\text { Wine } \\
\text { variety }\end{array}$} & \multirow[b]{3}{*}{ Origin } & \multicolumn{9}{|c|}{ Resveratrol contents (mg/L) } \\
\hline & & \multicolumn{3}{|c|}{ Hebei Huailai } & \multicolumn{3}{|c|}{ Ningxia Qingtongxia } & \multicolumn{3}{|c|}{ Xinjiang Changji } \\
\hline & & tRES & tPI & Total & tRES & tPI & Total & tRES & $\mathrm{tPI}$ & Total \\
\hline \multirow{4}{*}{$\begin{array}{l}\text { Red } \\
\text { wine }\end{array}$} & $\begin{array}{c}\text { Cabernet Sauvignon } \\
(\text { fresh })\end{array}$ & 1.95 & 2.86 & 4.81 & 2.19 & 3.05 & 5.24 & 2.03 & 2.66 & 4.69 \\
\hline & $\begin{array}{c}\text { Cabernet Sauvignon } \\
\text { (aging) }\end{array}$ & 1.62 & 2.99 & 4.61 & 1.85 & 3.18 & 5.03 & 1.72 & 2.73 & 4.45 \\
\hline & Merlot（fresh） & 2.01 & 3.27 & 5.28 & 2.87 & 3.92 & 6.79 & 1.98 & 3.01 & 4.99 \\
\hline & Merlot（aging） & 1.74 & 3.33 & 5.17 & 2.48 & 4.23 & 6.71 & 1.73 & 3.14 & 4.87 \\
\hline $\begin{array}{l}\text { White } \\
\text { wine }\end{array}$ & Chardonnay & 0.50 & n.d. & 0.50 & 0.84 & n.d. & 0.84 & 0.21 & 0.23 & 0.44 \\
\hline
\end{tabular}

note: tRES means trans-resveratrol,tPI means trans piceid.

As known,the resveratrol contents in wine is mainly depend on the grape cultivar, climate conditions of the regions: like humidity,temperatures and sunshine, and eaposure to fungal infection, fermentation techniques and so on. In the three regions, Resveratrol concentration in Ningxia wine was found the highest followed by Hebei wines, least Xinjiang wines. Although previous studies found more rainfall during maturation of grape berries could promote resveratrol accumulation in grape skins, and lower temperatures could inhibit synthesis of resveratrol, however both Ningxia Qingytongxia and Xinjiang Changji has less rainfall and larger temperatures differences between day and night, while Hebei Huailai has a higher average day temperature, more rainfall. That means the results were not completely dependent on rainfall or low temperature alone. It suggested that accumulation of resveratrol in grpes was complicated, might be influenced by combined climatic factors like sunshine time, humidity, temperatures difference between day and night, soil types, vintages and irrigation types.

For higher trans-piceid amount than trans- resveratrol in wines, it was not in agreement with some studies, in this study, trans-piceid amount was obviously higher than trans- resveratrol in almost all wines. Some of soil bacterium might help to transform resveratrol into piceid in the three regions ${ }^{[8]}$.We also observed the total resveratrol concentration in Merlot wines were higher than in Cabernet Sauvignon wines. Which was in accordence with others studies. It was still not very clearly about the accumulation machnism of resveratrol in different grape varieties, but probably they had different synthetic pathways of resveratrol in different grapes catalysed by the stilbene synthase. Because several STS genes showed different expression behavior as response to a biotic stresses ${ }^{[9]}$. And Zeng Qin found a resveratrol-producing endophytic fungus Alternaria sp. isolated from merlot grapes had a high capacity for yielding more resveratrols ${ }^{[10]}$.

Compared the fresh wines with aged wines,more trans-resveratrol was observed in fresh wines than in aged wines, which suggested that some of free resveratrol could be transferred into bound state resveratrol or glycosylated resveratrol during aging. Therefore the trans-piceid contents increased with aging process. However the total resveratrol content changed little, also showed that alcohol provided a stable environment for resvertrol during aging, or the extraction of resveratrol finished at the end of alcoholic fermentation

From table1, resveratrol contents in red wines were hugely higher than those in white wines, which indicated that differences in enological practices could lead to different results. Resveratrol is mainly accumulated in skins and seeds, during red wine fermentation, long time extraction of skins increaseed 
the transfermation of resveratrol from skins and seeds into the must with assitant of alcohols, while white wine feremented without grape skins ${ }^{[11]}$.

\section{Conclusions}

Resvertrol contents in wines is affected by some factors,including grapes virieties,grape regions,aging process,fermentation techniques and so on. The paper compared some virieties of wines in three main geographical regions in China. The resluts showed that resvertrol contents in Merlot wines was higher than in Cabernet Sauvignon wines, and those in Ningxia Qingtongxia region wines was the highest, follwed by Hebei Huailai wines, least Xinjiang Changji wines. And the contents in red wines was much higher than white wines. There was no significant difference of total resvertrol contents between fresh wine and aged wines. Totally, the highest resvertrol amount was $6.79 \mathrm{mg} / \mathrm{L}$, found in fresh merlot red wine in Ningxia Qingtongxia region in China. While least resvertrol amount was $0.44 \mathrm{mg} / \mathrm{L}$ found in Chardonnay white wine from Xinjiang Changji.

\section{Acknowledgements}

The work was supported by Transformation and Promotion Program of Agricultural Scientific and Technological Achievements of Tianjin ( 201502130).

\section{References}

[1] Fremont, Lucie, Biological effects of resveratrol, Life Sciences. 66,(2000) 663-673.

[2] Roy, H., Lundy, S., Resveratrol, Pennington Nutrition Series, 7(2005).

[3] Carter LG, D'Orazio JA, Pearson KJ, Resveratrol and cancer: focus on in vivo evidence, Endocr. Relat. Cancer. 21 (3) (2014): R209-25.

[4] Athar M., Back J.H., Tang X., Kim K.H., Kopelovich L, Bickers DR, Kim AL. Resveratrol: a review of preclinical studies for human cancer prevention, Toxicol. Appl. Pharmacol. 224 (3) (2007): 274-83.

[5] Wang L., et.,al, Natural product agonists of peroxisome proliferator-activated receptor gamma (PPAR $\gamma$ ): a review. Biochem Pharmacol. 92(2014): 73.

[6] Baliga M.S., Katiyar S.K.,Chemoprevention of photocarcinogenesis by selected dietary botanicals, Photochemical \& Photobiological Sciences. 5 (2)(2006): 243-53.

[7] Anekonda T.S., Resveratrol--a boon for treating Alzheimer's disease?. Brain Res Rev 52 (2)(2006): 316-26.

[8] Cichewicz R.H., Kouzi S.A., Kouzi,Biotransformation of resveratrol to piceid by bacillus cereus, J. Nat. Prod. 61 (10) (1998): 1313-4.

[9] Preisig-Muller R,et., al,Characterization of a pine multigene family containing elicitor responsive stilbene synthase genes, Plant Mol Bio. 139 (1999):221-222.

[10] Qin Zeng, JunLing Shi, Yanlin Liu, Isolation and Identification of a Resveratrol-Producing Endophytic Fungus from Grape, Food science. (33)13(2012):167-170.In Chinese.

[11] Monagas M.,Bartolome B.,Gomez-Cordoves C., Evolution of the polyphenols in red wine from Vitis ViniferaL during aging in the bottle. II .Non-anthocyanin phenolic compounds, Eur Food Res Techno 1220(2005):331-334. 